\title{
Antimicrobial resistance profiles of Shiga toxin-producing Escherichia coli 0157 and Non-0157 recovered from domestic farm animals in rural communities in Northwestern Mexico
}

\author{
Bianca A. Amézquita-López , Beatriz Quiñones ${ }^{2^{*}}$ (1), Marcela Soto-Beltrán ${ }^{1}$, Bertram G. Lee², Jaszemyn C. Yambao²,
} Ofelia Y. Lugo-Melchor ${ }^{3}$ and Cristóbal Chaidez ${ }^{4^{*}}$

\begin{abstract}
Background: Antimicrobial resistance in Shiga toxin-producing Escherichia coli (STEC) O157 and non-O157 is a matter of increasing concern. The aim of the present study was to investigate the antimicrobial resistance profiles of STEC O157 and non-O157 recovered from feces of domestic farm animals in the agricultural Culiacan Valley in Northwestern Mexico.

Findings: All of the examined STEC strains showed susceptibility to five antimicrobials, ceftazidime, ceftriaxone, ciprofloxacin, nalidixic acid, and trimethoprim-sulfamethoxazole. However, resistance to the four antimicrobials, ampicillin, cephalothin, chloramphenicol, and kanamycin was commonly observed. Interestingly, non-susceptibility to cephalothin was predominant among the examined STEC strains, corresponding to $85 \%$ (22/26) of the O157:H7 from cattle, sheep and chicken and $73 \%$ (24/33) of the non-0157 strains from cattle and sheep. Statistical analyses revealed that resistance to ampicillin was significantly correlated to $38 \%$ (10/26) of STEC O157:H7 strains from multiple animal sources. Another significant correlation was found between serotype, source, and antimicrobial resistance; all of the O20:H4 strains, recovered from sheep, were highly resistant to tetracycline. Multidrug resistance profiles were identified in $42 \%$ (22/53) of the non-susceptible STEC strains with clinically-relevant serotypes O8:H9, O75:H8, O146:H21, and O157:H7.

Conclusions: STEC O157 and non-O157 strains, recovered from domestic farm animals in the Culiacan Valley, exhibited resistance to classes of antimicrobials commonly used in Mexico, such as aminoglycosides, tetracyclines, cephalosporins and penicillin but were susceptible to fluoroquinolones, quinolones, and sulfonamides. These findings provide fundamental information that would aid in the surveillance of antimicrobial resistance in an important agricultural region in Northwestern Mexico.
\end{abstract}

Keywords: Antimicrobial resistance, Antibiotics, Domestic farm animals, Shiga toxin, Escherichia coli O157:H7, Escherichia coli non-O157, Mexico

\footnotetext{
*Correspondence: beatriz.quinones@ars.usda.gov; chaqui@ciad.mx

${ }^{2}$ U.S. Department of Agriculture/Agricultural Research Service, Western Regional Research Center, Produce Safety and Microbiology Research Unit, 800 Buchanan Street, Albany, CA 94710, USA

${ }^{4}$ Research Center in Food \& Development (CIAD), Food Safety National Research Laboratory (LANIIA), Carretera a El Dorado Km5.5, Col. Campo El Diez, 80110 Culiacan, Sinaloa, Mexico

Full list of author information is available at the end of the article
}

(c) 2016 Amézquita-López et al. Open Access This article is distributed under the terms of the Creative Commons Attribution 4.0 International License (http://creativecommons.org/licenses/by/4.0/), which permits unrestricted use, distribution, and reproduction in any medium, provided you give appropriate credit to the original author(s) and the source, provide a link to the Creative Commons license, and indicate if changes were made. The Creative Commons Public Domain Dedication waiver (http://creativecommons.org/publicdomain/zero/1.0/) applies to the data made available in this article, unless otherwise stated. 


\section{Introduction}

Shiga toxin-producing Escherichia coli (STEC) is a foodborne pathogen and causes severe gastroenteritis, hemorrhagic colitis, and the life-threatening hemolyticuremic syndrome (HUS) in humans [1]. Serotype O157:H7 has been implicated in most outbreaks [1]; however, other non-O157 serotypes have been associated with severe human infections worldwide [1-3]. Recently, several reports have documented a significant increase of antimicrobial resistance in STEC O157:H7 and non-O157:H7 strains $[4,5]$, and antibiotic resistance of E. coli in Mexico has increased over the years [6]. Inappropriate usages of antibiotics for treating human and plant diseases and for promoting food-animal growth are proposed to contribute to antimicrobial resistance among bacteria populations [6-9]. Moreover, the use of antimicrobials to treat STEC infections is controversial since they can induce Shiga toxin (Stx) production, resulting in HUS in humans [10-12]. However, other studies have suggested that if some classes of antimicrobials are administered early during the infection, STEC disease progression to the HUS could be prevented [10,13,14].

STEC strains have been recovered from a variety of animals, and cattle are considered the major reservoir for STEC strains $[1,15,16]$. Recent evidence has indicated that small domestic ruminants are also relevant STEC reservoirs $[16,17]$. Given that animals act as reservoirs of STEC that could potentially be transmitted to humans, thru direct or indirect contact, or via the food chain, the present study examined antimicrobial susceptibility in STEC O157 and non-O157 strains, recovered from feces of domestic farm animals [16]. The domestic farm animals were raised in small rural communities within the agricultural Culiacan Valley in Northwestern Mexico. The results indicated that STEC O157 and nonO157 strains exhibited resistance to aminoglycosides, tetracyclines, cephalosporins and penicillins, antimicrobials commonly used in Mexico [18-20]. However, all examined STEC strains were susceptible to fluoroquinolones, quinolones, and sulfonamides, agents that can induce Stx production $[10,12,21]$. These findings provide fundamental information that would aid in the surveillance of antimicrobial resistance patterns in an important agricultural region in Northwestern Mexico.

\section{Materials and methods}

\section{Bacterial strains and growth conditions}

A total of 59 STEC O157:H7 and non-O157 strains were isolated from domestic animal feces in small rural farms, near agricultural fields in the Culiacan Valley, Northwestern Mexico [16, 22]. The source, serotype and virulence potential of the tested STEC strains were previously characterized [16, 22]. STEC strains were routinely grown at $37^{\circ} \mathrm{C}$ on trypticase soy agar (Bioxon, Mexico City, Mexico) under aerobic conditions.

\section{Antimicrobial susceptibility testing}

The Kirby-Bauer disk diffusion method was performed to test 15 antimicrobials, representing 11 distinct classes (see Additional file 1), which are commonly used in Mexico for animal food production and human infection treatments [18-20]. Inoculums from each STEC strain were grown aerobically in $5 \mathrm{~mL}$ Mueller-Hinton $(\mathrm{MH})$ broth (Bioxon, Mexico City, Mexico) and incubated at $37{ }^{\circ} \mathrm{C}$ to reach a turbidity equal to a McFarland 0.5 standard, according to guidelines provided by Clinical and Laboratory Standards Institute (CLSI) [23]. MH agar plates were surface inoculated with each STEC culture using sterile cotton swabs, and antimicrobial paper disks (BD Diagnostics, Mexico City, Mexico) were placed on surface of inoculated $\mathrm{MH}$ agar plates. After incubation at $37^{\circ} \mathrm{C}$ for $16-18 \mathrm{~h}$, the diameter of the zone of microbial growth inhibition around the antimicrobial disk was measured in millimeters. E. coli ATCC 25922 (American Type Culture Collection, Manassas, VA) was used as a positive control for antimicrobial susceptibility. The minimum inhibitory concentration (MIC) was then determined according to the interpretive criteria established by CLSI to classify the STEC strains as sensitive, intermediate, or resistant to the tested antimicrobial agent [23].

\section{Statistical analysis}

Statistical differences were determined by performing the Fisher's exact test with the R Statistical Software (version 3.0.1; R Foundation for Statistical Computing, Vienna, Austria) [24]. A $p$-value $\leq 0.01$ was considered statistically significant.

\section{Results}

Susceptibility to all tested 15 antimicrobials was observed in $3 \%(1 / 26)$ of the O157:H7 cattle strains and $15 \%(5 / 33)$ non-O157 from cattle and sheep. All of the examined STEC strains showed susceptibility to five antimicrobials, CAZ, CIP, CRO, NAL, and SXT (Tables 1 and 2), and approximately $90 \%$ of the tested STEC strains showed susceptibility to AMC, AMK, CFP, GEN, IPM and TET. By contrast, non-susceptibility to the four antimicrobials, AMP, CEF, CHL, and KAN was commonly observed, and in particular, non-susceptibility to CEF, including intermediate and resistant categories, was predominant among the examined STEC strains, corresponding to $85 \%(22 / 26)$ of the O157:H7 (Table 1) and $73 \%(24 / 33)$ of the non-O157 strains (Table 2). Resistance to AMP was significantly correlated to $38 \%(10 / 26)$ of the $\mathrm{O} 157: \mathrm{H} 7 \quad$ strains $\quad(p$-value $=0.0107) . \quad$ A statistically significant correlation was found between serotype, 
Table 1 Antimicrobial MIC Values of STEC 0157:H7 strains examined in this study

\begin{tabular}{|c|c|c|c|c|c|c|c|c|c|c|c|c|c|c|c|c|c|}
\hline \multirow[t]{2}{*}{ Serotype } & \multirow[t]{2}{*}{ Strain } & \multirow[t]{2}{*}{ Source ${ }^{a}$} & \multicolumn{15}{|c|}{ Antimicrobial MIC Values $(\mu \mathrm{g} / \mathrm{mL})$} \\
\hline & & & $\mathrm{AMC}$ & AMK & AMP & CAZ & CEF & CFP & $\mathrm{CHL}$ & CIP & CRO & GEN & IPM & KAN & NAL & SXT & TET \\
\hline \multirow[t]{26}{*}{ O157:H7 } & RM8744 & Cattle & $\leq 8 / 4$ & $\leq 16$ & $\leq 8$ & $\leq 4$ & $\geq 32^{b}$ & $32^{c}$ & $16^{c}$ & $\leq 1$ & $\leq 1$ & $\leq 4$ & $\leq 1$ & $\leq 16$ & $\leq 16$ & $\leq 2 / 38$ & $\leq 4$ \\
\hline & RM8753 & Sheep & $\leq 8 / 4$ & $32^{c}$ & $\leq 8$ & $\leq 4$ & $\geq 32^{b}$ & $\leq 16$ & $16^{\mathrm{c}}$ & $\leq 1$ & $\leq 1$ & $\leq 4$ & $\leq 1$ & $32^{c}$ & $\leq 16$ & $\leq 2 / 38$ & $\leq 4$ \\
\hline & RM8754 & Cattle & $\leq 8 / 4$ & $\leq 16$ & $\leq 8$ & $\leq 4$ & $\leq 8$ & $\leq 16$ & $\leq 8$ & $\leq 1$ & $\leq 1$ & $\leq 4$ & $\leq 1$ & $\leq 16$ & $\leq 16$ & $\leq 2 / 38$ & $\leq 4$ \\
\hline & RM8759 & Sheep & $\leq 8 / 4$ & $\leq 16$ & $\leq 8$ & $\leq 4$ & $\geq 32^{b}$ & $\leq 16$ & $\leq 8$ & $\leq 1$ & $\leq 1$ & $\leq 4$ & $\leq 1$ & $\leq 16$ & $\leq 16$ & $\leq 2 / 38$ & $\leq 4$ \\
\hline & RM8767 & Cattle & $\leq 8 / 4$ & $\leq 16$ & $\leq 8$ & $\leq 4$ & $16^{c}$ & $\leq 16$ & $\leq 8$ & $\leq 1$ & $\leq 1$ & $\leq 4$ & $\leq 1$ & $\leq 16$ & $\leq 16$ & $\leq 2 / 38$ & $\leq 4$ \\
\hline & RM8768 & Cattle & $\leq 8 / 4$ & $\leq 16$ & $\leq 8$ & $\leq 4$ & $\geq 32^{\mathrm{b}}$ & $\leq 16$ & $16^{c}$ & $\leq 1$ & $\leq 1$ & $\leq 4$ & $\leq 1$ & $\leq 16$ & $\leq 16$ & $\leq 2 / 38$ & $\leq 4$ \\
\hline & RM8769 & Cattle & $\leq 8 / 4$ & $\leq 16$ & $\leq 8$ & $\leq 4$ & $\leq 8$ & $\leq 16$ & $16^{c}$ & $\leq 1$ & $\leq 1$ & $\leq 4$ & $\leq 1$ & $\leq 16$ & $\leq 16$ & $\leq 2 / 38$ & $\leq 4$ \\
\hline & RM8771 & Cattle & $\leq 8 / 4$ & $\leq 16$ & $\leq 8$ & $\leq 4$ & $\leq 8$ & $\leq 16$ & $16^{c}$ & $\leq 1$ & $\leq 1$ & $\leq 4$ & $\leq 1$ & $\leq 16$ & $\leq 16$ & $\leq 2 / 38$ & $\leq 4$ \\
\hline & RM8781 & Sheep & $\leq 8 / 4$ & $\leq 16$ & $\leq 8$ & $\leq 4$ & $16^{c}$ & $\leq 16$ & $\leq 8$ & $\leq 1$ & $\leq 1$ & $\leq 4$ & $\leq 1$ & $32^{c}$ & $\leq 16$ & $\leq 2 / 38$ & $\leq 4$ \\
\hline & RM8920 & Sheep & $\leq 8 / 4$ & $\leq 16$ & $\leq 8$ & $\leq 4$ & $16^{c}$ & $\leq 16$ & $16^{c}$ & $\leq 1$ & $\leq 1$ & $\leq 4$ & $\leq 1$ & $\leq 16$ & $\leq 16$ & $\leq 2 / 38$ & $\leq 4$ \\
\hline & RM8921 & Sheep & $\leq 8 / 4$ & $\leq 16$ & $\leq 8$ & $\leq 4$ & $\geq 32^{b}$ & $\leq 16$ & $16^{\mathrm{c}}$ & $\leq 1$ & $\leq 1$ & $\leq 4$ & $\leq 1$ & $32^{c}$ & $\leq 16$ & $\leq 2 / 38$ & $\leq 4$ \\
\hline & RM8922 & Cattle & $\leq 8 / 4$ & $\leq 16$ & $\leq 8$ & $\leq 4$ & $16^{c}$ & $\leq 16$ & $\leq 8$ & $\leq 1$ & $\leq 1$ & $\leq 4$ & $\leq 1$ & $\leq 16$ & $\leq 16$ & $\leq 2 / 38$ & $\leq 4$ \\
\hline & RM9450 & Sheep & $\leq 8 / 4$ & $\leq 16$ & $\leq 8$ & $\leq 4$ & $\geq 32^{b}$ & $\leq 16$ & $\leq 8$ & $\leq 1$ & $\leq 1$ & $\leq 4$ & $\leq 1$ & $\leq 16$ & $\leq 16$ & $\leq 2 / 38$ & $\leq 4$ \\
\hline & RM9451 & Sheep & $\leq 8 / 4$ & $\leq 16$ & $\leq 8$ & $\leq 4$ & $16^{c}$ & $\leq 16$ & $\geq 32^{b}$ & $\leq 1$ & $\leq 1$ & $\leq 4$ & $\leq 1$ & $\geq 64^{b}$ & $\leq 16$ & $\leq 2 / 38$ & $\geq 16^{b}$ \\
\hline & RM9452 & Sheep & $\leq 8 / 4$ & $\leq 16$ & $\leq 8$ & $\leq 4$ & $\geq 32^{b}$ & $\leq 16$ & $16^{c}$ & $\leq 1$ & $\leq 1$ & $\leq 4$ & $\leq 1$ & $\leq 16$ & $\leq 16$ & $\leq 2 / 38$ & $\leq 4$ \\
\hline & RM9453 & Sheep & $\leq 8 / 4$ & $32^{c}$ & $\leq 8$ & $\leq 4$ & $\geq 32^{\mathrm{b}}$ & $\leq 16$ & $16^{c}$ & $\leq 1$ & $\leq 1$ & $\leq 4$ & $\leq 1$ & $32^{c}$ & $\leq 16$ & $\leq 2 / 38$ & $\leq 4$ \\
\hline & RM9454 & Cattle & $\leq 8 / 4$ & $\leq 16$ & $\geq 32^{b}$ & $\leq 4$ & $16^{c}$ & $\leq 16$ & $\leq 8$ & $\leq 1$ & $\leq 1$ & $\leq 4$ & $\leq 1$ & $\leq 16$ & $\leq 16$ & $\leq 2 / 38$ & $\leq 4$ \\
\hline & RM9455 & Cattle & $\leq 8 / 4$ & $\leq 16$ & $\geq 32^{\mathrm{b}}$ & $\leq 4$ & $16^{c}$ & $\leq 16$ & $\leq 8$ & $\leq 1$ & $\leq 1$ & $\leq 4$ & $\leq 1$ & $\leq 16$ & $\leq 16$ & $\leq 2 / 38$ & $\leq 4$ \\
\hline & RM9456 & Cattle & $\leq 8 / 4$ & $\leq 16$ & $\geq 32^{\mathrm{b}}$ & $\leq 4$ & $16^{c}$ & $\leq 16$ & $16^{c}$ & $\leq 1$ & $\leq 1$ & $\leq 4$ & $\leq 1$ & $32^{c}$ & $\leq 16$ & $\leq 2 / 38$ & $\leq 4$ \\
\hline & RM9457 & Cattle & $\leq 8 / 4$ & $\leq 16$ & $\geq 32^{\mathrm{b}}$ & $\leq 4$ & $16^{c}$ & $\leq 16$ & $\leq 8$ & $\leq 1$ & $\leq 1$ & $\leq 4$ & $\leq 1$ & $\leq 16$ & $\leq 16$ & $\leq 2 / 38$ & $\leq 4$ \\
\hline & RM9458 & Chicken & $\leq 8 / 4$ & $\leq 16$ & $\geq 32^{b}$ & $\leq 4$ & $16^{c}$ & $\leq 16$ & $\leq 8$ & $\leq 1$ & $\leq 1$ & $\leq 4$ & $\leq 1$ & $\leq 16$ & $\leq 16$ & $\leq 2 / 38$ & $\leq 4$ \\
\hline & RM9459 & Chicken & $\leq 8 / 4$ & $\leq 16$ & $\geq 32^{b}$ & $\leq 4$ & $16^{c}$ & $\leq 16$ & $\leq 8$ & $\leq 1$ & $\leq 1$ & $\leq 4$ & $\leq 1$ & $\leq 16$ & $\leq 16$ & $\leq 2 / 38$ & $\leq 4$ \\
\hline & RM9460 & Cattle & $\leq 8 / 4$ & $\leq 16$ & $\geq 32^{\mathrm{b}}$ & $\leq 4$ & $16^{c}$ & $\leq 16$ & $\leq 8$ & $\leq 1$ & $\leq 1$ & $\leq 4$ & $\leq 1$ & $\leq 16$ & $\leq 16$ & $\leq 2 / 38$ & $\leq 4$ \\
\hline & RM9461 & Cattle & $\leq 8 / 4$ & $\leq 16$ & $\geq 32^{\mathrm{b}}$ & $\leq 4$ & $16^{c}$ & $\leq 16$ & $\leq 8$ & $\leq 1$ & $\leq 1$ & $\leq 4$ & $\leq 1$ & $\leq 16$ & $\leq 16$ & $\leq 2 / 38$ & $\leq 4$ \\
\hline & RM9462 & Cattle & $16 / 8^{c}$ & $32^{c}$ & $\geq 32^{b}$ & $\leq 4$ & $16^{c}$ & $\leq 16$ & $\leq 8$ & $\leq 1$ & $\leq 1$ & $\leq 4$ & $\leq 1$ & $32^{c}$ & $\leq 16$ & $\leq 2 / 38$ & $\leq 4$ \\
\hline & RM9463 & Sheep & $\leq 8 / 4$ & $\leq 16$ & $\geq 32^{\mathrm{b}}$ & $\leq 4$ & $\leq 8$ & $\leq 16$ & $\leq 8$ & $\leq 1$ & $\leq 1$ & $\leq 4$ & $\leq 1$ & $\leq 16$ & $\leq 16$ & $\leq 2 / 38$ & $\leq 4$ \\
\hline
\end{tabular}

${ }^{a}$ Domestic animal samples were collected from distinct regions in the Culiacan Valley, Sinaloa, Mexico [16]

${ }^{\mathrm{b}} \mathrm{MIC}$ value in bold indicates resistance to the tested antimicrobial, according to CLSI guidelines [23]

${ }^{\mathrm{c}}$ MIC value indicates an intermediate susceptibility to the tested antimicrobial, according to CLSI guidelines [23]

source, and antimicrobial resistance; all of the O20:H4 strains, recovered from sheep, were resistant to TET $(p$-value $=0.0001)$, accounting for $75 \%(3 / 4)$ of the TET resistant STEC strains. No other correlation was found for other non-O157 serotypes and antimicrobials tested.

Correlation analysis of non-susceptibility to more than one antimicrobial, belonging to different classes, indicated that $37 \%(22 / 59)$ of the examined STEC strains exhibited resistance to both the aminoglycoside KAN and to the $1^{\text {st }}$ generation-cephalosporin CEF, resulting in a statistically significant correlation ( $p$-value $=0.0001)$ (Tables 1 and 2 ). Other observations were that $93 \%(14 / 15)$ and $80 \%$ (24/30) of the AMP and CHL non-susceptible strains, respectively, also showed non-susceptibility to CEF; however, these associations were not found to be statistically significant. The analyses revealed 19 distinct antimicrobial resistant profiles, and 12 were classified as multidrug resistant profiles (Table 3), indicating nonsusceptibility to more than 3 agents in different classes [25]. These multidrug resistance profiles were observed in $42 \%(22 / 53)$ of the non-susceptible STEC strains with clinically-relevant serotypes O75:H8, O146:H21, O8:H9, and O157:H7 (Table 3). The analysis also revealed that a particular antimicrobial resistance profile was not significantly correlated with animal source or STEC serotype.

\section{Discussion}

Many factors have been proposed to contribute to antimicrobial resistance in enteric bacterial pathogens, such as the inappropriate prescription and use of antibiotics in the public, private, and agricultural sectors [6-9]. Moreover, data from surveillance programs in Mexico have reported an apparent increase in antimicrobial 
Table 2 Antimicrobial MIC Values of STEC non-O157 strains examined in this study

\begin{tabular}{|c|c|c|c|c|c|c|c|c|c|c|c|c|c|c|c|c|c|}
\hline \multirow[t]{2}{*}{ Serotype ${ }^{a}$} & \multirow[t]{2}{*}{ Strain } & \multirow[t]{2}{*}{ Source $^{b}$} & \multicolumn{15}{|c|}{ Antimicrobial MIC Values $(\mu \mathrm{g} / \mathrm{mL})$} \\
\hline & & & AMC & AMK & AMP & CAZ & CEF & CFP & $\mathrm{CHL}$ & CIP & CRO & GEN & IPM & KAN & NAL & SXT & TET \\
\hline O8:NT & RM8766 & Sheep & $\leq 8 / 4$ & $\leq 16$ & $\leq 8$ & $\leq 4$ & $\leq 8$ & $\leq 16$ & $\leq 8$ & $\leq 1$ & $\leq 1$ & $\leq 4$ & $\leq 1$ & $\leq 16$ & $\leq 16$ & $\leq 2 / 38$ & $\leq 4$ \\
\hline \multirow[t]{5}{*}{$\mathrm{O} 8 \mathrm{H} 19$} & RM8772 & Cattle & $\leq 8 / 4$ & $32^{d}$ & $\leq 8$ & $\leq 4$ & $16^{\mathrm{d}}$ & $\leq 16$ & $16^{d}$ & $\leq 1$ & $\leq 1$ & $\leq 4$ & $\leq 1$ & $32^{d}$ & $\leq 16$ & $\leq 2 / 38$ & $\leq 4$ \\
\hline & RM8773 & Cattle & $\leq 8 / 4$ & $\leq 16$ & $\leq 8$ & $\leq 4$ & $\leq 8$ & $\leq 16$ & $\leq 8$ & $\leq 1$ & $\leq 1$ & $\leq 4$ & $\leq 1$ & $\leq 16$ & $\leq 16$ & $\leq 2 / 38$ & $\leq 4$ \\
\hline & RM8774 & Cattle & $\leq 8 / 4$ & $\leq 16$ & $\leq 8$ & $\leq 4$ & $16^{d}$ & $\leq 16$ & $16^{d}$ & $\leq 1$ & $\leq 1$ & $\leq 4$ & $\leq 1$ & $\leq 16$ & $\leq 16$ & $\leq 2 / 38$ & $\leq 4$ \\
\hline & RM8775 & Cattle & $\leq 8 / 4$ & $\leq 16$ & $\leq 8$ & $\leq 4$ & $\geq 32^{c}$ & $\leq 16$ & $16^{d}$ & $\leq 1$ & $\leq 1$ & $\leq 4$ & $\leq 1$ & $32^{d}$ & $\leq 16$ & $\leq 2 / 38$ & $\leq 4$ \\
\hline & RM8776 & Cattle & $\leq 8 / 4$ & $\leq 16$ & $\leq 8$ & $\leq 4$ & $\geq 32^{c}$ & $\leq 16$ & $\geq 32^{c}$ & $\leq 1$ & $\leq 1$ & $\leq 4$ & $\leq 1$ & $32^{d}$ & $\leq 16$ & $\leq 2 / 38$ & $\leq 4$ \\
\hline O15:NT & RM8747 & Cattle & $\geq 32 / 16^{c}$ & $\leq 16$ & $\geq 32^{c}$ & $\leq 4$ & $\geq 32^{c}$ & $\leq 16$ & $16^{d}$ & $\leq 1$ & $\leq 1$ & $\leq 4$ & $\leq 1$ & $\leq 16$ & $\leq 16$ & $\leq 2 / 38$ & $\leq 4$ \\
\hline \multirow[t]{3}{*}{$\mathrm{O} 2 \mathrm{O}: \mathrm{H} 4$} & RM8749 & Sheep & $\leq 8 / 4$ & $\leq 16$ & $\geq 32^{c}$ & $\leq 4$ & $16^{d}$ & $\leq 16$ & $\geq 32^{c}$ & $\leq 1$ & $\leq 1$ & $\leq 4$ & $\leq 1$ & $\leq 16$ & $\leq 16$ & $\leq 2 / 38$ & $\geq 16^{c}$ \\
\hline & RM8750 & Sheep & $\leq 8 / 4$ & $\leq 16$ & $16^{d}$ & $\leq 4$ & $\geq 32^{c}$ & $\leq 16$ & $\leq 8$ & $\leq 1$ & $\leq 1$ & $\leq 4$ & $\leq 1$ & $\leq 16$ & $\leq 16$ & $\leq 2 / 38$ & $\geq 16^{c}$ \\
\hline & RM8751 & Sheep & $\leq 8 / 4$ & $\leq 16$ & $\leq 8$ & $\leq 4$ & $16^{d}$ & $\leq 16$ & $\leq 8$ & $\leq 1$ & $\leq 1$ & $\leq 4$ & $\leq 1$ & $32^{d}$ & $\leq 16$ & $\leq 2 / 38$ & $\geq 16^{c}$ \\
\hline O73:NT & RM8748 & Cattle & $\leq 8 / 4$ & $\leq 16$ & $\leq 8$ & $\leq 4$ & $16^{d}$ & $\leq 16$ & $16^{d}$ & $\leq 1$ & $\leq 1$ & $\leq 4$ & $\leq 1$ & $\leq 16$ & $\leq 16$ & $\leq 2 / 38$ & $\leq 4$ \\
\hline \multirow[t]{2}{*}{ O73:H4 } & RM8745 & Sheep & $\leq 8 / 4$ & $\leq 16$ & $\leq 8$ & $\leq 4$ & $\geq 32^{c}$ & $\leq 16$ & $\leq 8$ & $\leq 1$ & $\leq 1$ & $\leq 4$ & $\leq 1$ & $32^{d}$ & $\leq 16$ & $\leq 2 / 38$ & $\leq 4$ \\
\hline & RM8746 & Sheep & $\leq 8 / 4$ & $\leq 16$ & $\leq 8$ & $\leq 4$ & $\geq 32^{c}$ & $\leq 16$ & $16^{d}$ & $\leq 1$ & $\leq 1$ & $\leq 4$ & $\leq 1$ & $32^{d}$ & $\leq 16$ & $\leq 2 / 38$ & $\leq 4$ \\
\hline \multirow[t]{12}{*}{$\mathrm{O} 75: \mathrm{H} 8$} & RM8752 & Sheep & $\leq 8 / 4$ & $\leq 16$ & $16^{d}$ & $\leq 4$ & $\geq 32^{c}$ & $\leq 16$ & $\leq 8$ & $\leq 1$ & $\leq 1$ & $\leq 4$ & $\leq 1$ & $32^{d}$ & $\leq 16$ & $\leq 2 / 38$ & $\leq 4$ \\
\hline & RM8760 & Sheep & $\leq 8 / 4$ & $\leq 16$ & $\leq 8$ & $\leq 4$ & $\leq 8$ & $\leq 16$ & $16^{d}$ & $\leq 1$ & $\leq 1$ & $\leq 4$ & $\leq 1$ & $\leq 16$ & $\leq 16$ & $\leq 2 / 38$ & $\leq 4$ \\
\hline & RM8763 & Sheep & $\leq 8 / 4$ & $\leq 16$ & $\leq 8$ & $\leq 4$ & $\leq 8$ & $\leq 16$ & $\leq 8$ & $\leq 1$ & $\leq 1$ & $\leq 4$ & $\leq 1$ & $\leq 16$ & $\leq 16$ & $\leq 2 / 38$ & $\leq 4$ \\
\hline & RM8764 & Sheep & $\leq 8 / 4$ & $\leq 16$ & $\leq 8$ & $\leq 4$ & $\leq 8$ & $\leq 16$ & $16^{\mathrm{d}}$ & $\leq 1$ & $\leq 1$ & $\leq 4$ & $\leq 1$ & $\leq 16$ & $\leq 16$ & $\leq 2 / 38$ & $\leq 4$ \\
\hline & RM8765 & Sheep & $\leq 8 / 4$ & $\leq 16$ & $\leq 8$ & $\leq 4$ & $16^{d}$ & $\leq 16$ & $\leq 8$ & $\leq 1$ & $\leq 1$ & $\leq 4$ & $\leq 1$ & $32^{d}$ & $\leq 16$ & $\leq 2 / 38$ & $\leq 4$ \\
\hline & RM8778 & Sheep & $\leq 8 / 4$ & $\leq 16$ & $\leq 8$ & $\leq 4$ & $\geq 32^{c}$ & $\leq 16$ & $16^{d}$ & $\leq 1$ & $\leq 1$ & $\leq 4$ & $\leq 1$ & $32^{d}$ & $\leq 16$ & $\leq 2 / 38$ & $\leq 4$ \\
\hline & RM8779 & Sheep & $\leq 8 / 4$ & $\leq 16$ & $\leq 8$ & $\leq 4$ & $16^{d}$ & $\leq 16$ & $16^{d}$ & $\leq 1$ & $\leq 1$ & $\leq 4$ & $\leq 1$ & $\leq 16$ & $\leq 16$ & $\leq 2 / 38$ & $\leq 4$ \\
\hline & RM8780 & Sheep & $\leq 8 / 4$ & $\leq 16$ & $\leq 8$ & $\leq 4$ & $\leq 8$ & $\leq 16$ & $16^{d}$ & $\leq 1$ & $\leq 1$ & $\leq 4$ & $\leq 1$ & $\leq 16$ & $\leq 16$ & $\leq 2 / 38$ & $\leq 4$ \\
\hline & RM8923 & Cattle & $\leq 8 / 4$ & $\leq 16$ & $\leq 8$ & $\leq 4$ & $16^{d}$ & $\leq 16$ & $16^{d}$ & $\leq 1$ & $\leq 1$ & $\leq 4$ & $\leq 1$ & $32^{d}$ & $\leq 16$ & $\leq 2 / 38$ & $\leq 4$ \\
\hline & RM8929 & Sheep & $\leq 8 / 4$ & $\leq 16$ & $\leq 8$ & $\leq 4$ & $16^{d}$ & $\leq 16$ & $16^{d}$ & $\leq 1$ & $\leq 1$ & $\leq 4$ & $\geq 4^{c}$ & $32^{d}$ & $\leq 16$ & $\leq 2 / 38$ & $\leq 4$ \\
\hline & RM8930 & Sheep & $\leq 8 / 4$ & $\leq 16$ & $\leq 8$ & $\leq 4$ & $16^{d}$ & $\leq 16$ & $16^{d}$ & $\leq 1$ & $\leq 1$ & $\leq 4$ & $\leq 1$ & $32^{d}$ & $\leq 16$ & $\leq 2 / 38$ & $\leq 4$ \\
\hline & RM13865 & Cattle & $\leq 8 / 4$ & $\leq 16$ & $\geq 32^{c}$ & $\leq 4$ & $16^{d}$ & $\leq 16$ & $\leq 8$ & $\leq 1$ & $\leq 1$ & $\leq 4$ & $\leq 1$ & $32^{d}$ & $\leq 16$ & $\leq 2 / 38$ & $\leq 4$ \\
\hline \multirow[t]{2}{*}{ O111:H8 } & RM8755 & Sheep & $\leq 8 / 4$ & $\leq 16$ & $\leq 8$ & $\leq 4$ & $16^{d}$ & $\leq 16$ & $\leq 8$ & $\leq 1$ & $\leq 1$ & $\geq 16^{c}$ & $\leq 1$ & $\leq 16$ & $\leq 16$ & $\leq 2 / 38$ & $\leq 4$ \\
\hline & RM8916 & Sheep & $\leq 8 / 4$ & $\leq 16$ & $\leq 8$ & $\leq 4$ & $\geq 32^{c}$ & $\leq 16$ & $16^{d}$ & $\leq 1$ & $\leq 1$ & $\leq 4$ & $\leq 1$ & $\leq 16$ & $\leq 16$ & $\leq 2 / 38$ & $\leq 4$ \\
\hline O146:H8 & RM8762 & Sheep & $\leq 8 / 4$ & $\leq 16$ & $\leq 8$ & $\leq 4$ & $\leq 8$ & $\leq 16$ & $\leq 8$ & $\leq 1$ & $\leq 1$ & $\leq 4$ & $\leq 1$ & $\leq 16$ & $\leq 16$ & $\leq 2 / 38$ & $\leq 4$ \\
\hline \multirow[t]{4}{*}{ O146:H21 } & RM8756 & Sheep & $\leq 8 / 4$ & $\leq 16$ & $\leq 8$ & $\leq 4$ & $\leq 8$ & $\leq 16$ & $\leq 8$ & $\leq 1$ & $\leq 1$ & $\leq 4$ & $\leq 1$ & $\leq 16$ & $\leq 16$ & $\leq 2 / 38$ & $\leq 4$ \\
\hline & RM8757 & Sheep & $\leq 8 / 4$ & $\leq 16$ & $\leq 8$ & $\leq 4$ & $\leq 8$ & $\leq 16$ & $16^{\mathrm{d}}$ & $\leq 1$ & $\leq 1$ & $\leq 4$ & $\leq 1$ & $\leq 16$ & $\leq 16$ & $\leq 2 / 38$ & $\leq 4$ \\
\hline & RM8758 & Sheep & $\leq 8 / 4$ & $\leq 16$ & $\leq 8$ & $\leq 4$ & $16^{d}$ & $\leq 16$ & $16^{d}$ & $\leq 1$ & $\leq 1$ & $\leq 4$ & $\leq 1$ & $32^{d}$ & $\leq 16$ & $\leq 2 / 38$ & $\leq 4$ \\
\hline & RM8761 & Sheep & $\leq 8 / 4$ & $\leq 16$ & $\leq 8$ & $\leq 4$ & $16^{d}$ & $\leq 16$ & $\leq 8$ & $\leq 1$ & $\leq 1$ & $\leq 4$ & $\leq 1$ & $\leq 16$ & $\leq 16$ & $\leq 2 / 38$ & $\leq 4$ \\
\hline O168:NT & RM8917 & Cattle & $\leq 8 / 4$ & $\leq 16$ & $\leq 8$ & $\leq 4$ & $16^{d}$ & $\leq 16$ & $\leq 8$ & $\leq 1$ & $\leq 1$ & $\leq 4$ & $\leq 1$ & $32^{d}$ & $\leq 16$ & $\leq 2 / 38$ & $\leq 4$ \\
\hline
\end{tabular}

${ }^{\mathrm{a} N T}$, Non-typeable H-antigen

${ }^{b}$ Domestic animal samples were collected from distinct regions in the Culiacan Valley, Sinaloa, Mexico [16]

${ }^{\mathrm{c}} \mathrm{MIC}$ value indicates resistance to the tested antimicrobial, according to CLSI guidelines [23]

${ }^{\mathrm{d}} \mathrm{MIC}$ value indicates an intermediate susceptibility to the tested antimicrobial, according to CLSI guidelines [23]

resistance of $E$. coli over the years [6, 18]. In the present study, resistance to antimicrobials, belonging to classes commonly utilized in Mexico [18-20], was investigated in zoonotic STEC. The STEC strains were recovered from domestic farm animals in small rural communities that were adjacent to the agricultural Culiacan Valley, where the primary purpose of raising livestock is for local consumption [16]. The findings of this study provide a better understanding of resistance to antimicrobial agents in an important agricultural region in Mexico and will aid in the development of efficient and targeted intervention strategies.

The results from the present study demonstrated that zoonotic STEC O157 and non-O157, recovered from the Culiacan Valley, were resistant to antimicrobials belonging to classes such as aminoglycosides, beta lactams, 
Table 3 Antimicrobial resistance profiles identified in the STEC 0157 and non-O157 from different animal sources

\begin{tabular}{|c|c|c|}
\hline STEC Serotypes $(n)^{a}$ & Sources $^{b}$ & Resistance Profile \\
\hline O157:H7 (1) & Sheep & AMP \\
\hline O146:H21 (1), O157:H7 (4) & Sheep Cattle & CEF \\
\hline $\mathrm{O} 75: \mathrm{H} 8$ (3), O146:H21 (1), O157:H7 (2) & Sheep Cattle & $\mathrm{CHL}$ \\
\hline O157:H7 (7) & Cattle, Chicken & AMP, CEF \\
\hline O8:H19 (1), O73:NT (1), O75:H8 (1), O111:H8 (1), O157:H7 (3) & Sheep, Cattle & $\mathrm{CEF}, \mathrm{CHL}$ \\
\hline O111:H8 (1) & Sheep & CEF, GEN \\
\hline O157:H7 (1), O73:H4 (1), O75:H8 (1), O168:NT (1) & Sheep, Cattle & CEF, KAN \\
\hline O75:H8 (2) & Sheep, Cattle & AMP, CEF, KAN \\
\hline $\mathrm{O} 2 \mathrm{O}: \mathrm{H} 4(1)$ & Sheep & AMP, CEF, TET \\
\hline O157:H7 (1) & Cattle & CEF, CFP, CHL \\
\hline $\mathrm{O} 8: \mathrm{H} 19$ (2), O73:H4 (1), O75:H8 (3), O146:H21 (1), O157:H7 (1) & Sheep, Cattle & $\mathrm{CEF}, \mathrm{CHL}, \mathrm{KAN}$ \\
\hline $\mathrm{O} 20: \mathrm{H} 4(1)$ & Sheep & CEF, KAN, TET \\
\hline O157:H7 (1) & Cattle & $\mathrm{AMP}, \mathrm{CEF}, \mathrm{CHL}, \mathrm{KAN}$ \\
\hline $\mathrm{O} 20: \mathrm{H} 4(1)$ & Sheep & AMP, CEF, CHL, TET \\
\hline O75:H8 (1) & Sheep & $\mathrm{CEF}, \mathrm{CHL}, \mathrm{KAN}, \mathrm{IPM}$ \\
\hline O157:H7 (1) & Sheep & CEF, CHL, KAN, TET \\
\hline O15:NT (1) & Cattle & $\mathrm{AMC}, \mathrm{AMP}, \mathrm{CEF}, \mathrm{CHL}$ \\
\hline O8:H19 (1), O157:H7 (2) & Sheep, Cattle & $\mathrm{AMK}, \mathrm{CEF}, \mathrm{CHL}, \mathrm{KAN}$ \\
\hline O157:H7 (1) & Cattle & AMC, AMK, AMP, CEF, KAN \\
\hline
\end{tabular}

${ }^{a} \mathrm{NT}$, Non-typeable $\mathrm{H}$-antigen

${ }^{b}$ Domestic animal samples were collected from distinct regions in the Culiacan Valley, Sinaloa, Mexico [16]

carbapenem, cephalosporins, phenicols, and tetracyclines. In particular, resistance to CEF, a $1^{\text {st }}$-generation cephalosporin, was prominently detected in $78 \%(46 / 59)$ of the tested STEC O157 and non-O157 strains. Interestingly, the present study has demonstrated for the first time a significant correlation for AMP resistance in O157:H7 and TET resistance in O20:H4 zoonotic STEC strains recovered from Northwestern Mexico. In agreement with published findings on STECs recovered from foods in this geographical region [18], susceptibility was observed for sulfonamides, quinolones and fluoroquinolones in the recovered STEC strains. These agents have been found to induce Stx production in STEC strains [10,21], potentially increasing the risk of HUS. However, all STEC strains were susceptible to the $3^{\text {rd }}$-generation cephalosporin $\mathrm{CRO}$, which does not promote Stx production [21].

Classification of multidrug-resistance, based on recently published criteria [25], was observed in $42 \%$ $(22 / 53)$ of the non-susceptible STEC strains, harboring serotypes associated with human illness [2]. Multidrug resistance profiles, described in the present study, included classes of antimicrobials commonly used in Mexico, such as aminoglycosides, tetracyclines, cephalosporins, and penicillins [18-20], and these findings highlight the need for surveillance of the antimicrobial resistance patterns in enteric bacterial pathogens. Future work is aimed at further dissecting the genetic elements contributing to the acquisition and dissemination of the antimicrobial resistance genes in STEC strains recovered from agricultural regions in Northwestern Mexico.

\section{Additional file}

Additional file 1: Antimicrobial agents used in the present study. (DOCX $14 \mathrm{~kb}$ )

\section{Abbreviations}

AMC: Amoxicillin - clavulanic acid; AMK: Amikacin; AMP: Ampicillin: CAZ: Ceftazidime; CEF: Cephalothin; CFP: Cefoperazone;

CHL: Chloramphenicol; CIP: Ciprofloxacin; CLSI: Clinical and Laboratory Standards Institute; CRO: Ceftriaxone; GEN: Gentamicin; HUS: Hemolytic uremic syndrome; IPM: Imipenem; KAN: Kanamycin; MDR: Multi-drug resistant; $\mathrm{MH}$ : Mueller-Hinton; MIC: Minimum inhibitory concentration; NAL: Nalidixic acid; STEC: Shiga toxin-producing Escherichia coli; Stx: Shiga toxin; SXT: Trimethoprim-sulfamethoxazole; TET: Tetracycline.

\section{Competing interests}

The authors declare that they have no competing interests.

\section{Authors' contributions}

$B A A L, C C, B Q$, and OYLM conceived and designed the experiments. BQ, $B A A L, B L$ and $J Y$ were responsible for the acquisition and analysis of microbiological data and manuscript preparation. BQ, BL, BAAL, CC, MSB and OYLM contributed reagents/materials/analysis tools. All authors have read and approved the final manuscript.

\section{Acknowledgments}

This work was supported by the U.S. Department of Agriculture, Agricultural Research Service CRIS project number 5325-42000-047 and by a Postgraduate Studies Scholarship from The National Council of Science and Technology in 
Mexico (CONACyT grant \#234885) to BAAL. The authors would like to thank Lucía Tamayo and Célida Martínez (CIAD in Culiacan, Sinaloa, Mexico) for excellent technical assistance.

\section{Author details \\ ${ }^{1}$ Faculty of Chemical and Biological Sciences (FCQB), The Autonomous University of Sinaloa (UAS), Josefa Ortiz de Domínguez y Blvd. de las Américas S/N, Ciudad Universitaria, 80013 Culiacan, Sinaloa, México. ${ }^{2}$ U.S. Department of Agriculture/Agricultural Research Service, Western Regional Research Center, Produce Safety and Microbiology Research Unit, 800 Buchanan Street, Albany, CA 94710, USA. ${ }^{3}$ Centro de Investigación y Asistencia en Tecnología y Diseño del Estado de Jalisco (CIATEJ), Normalistas 800, Colinas de La Normal, 44270 Guadalajara, Jalisco, Mexico. ${ }^{4}$ Research Center in Food \& Development (CIAD), Food Safety National Research Laboratory (LANIIA), Carretera a El Dorado Km5.5, Col. Campo El Diez, 80110 Culiacan, Sinaloa, Mexico. \\ Received: 25 September 2015 Accepted: 18 December 2015 \\ Published online: 05 January 2016}

\section{References}

1. Gyles CL. Shiga toxin-producing Escherichia coli: an overview. J Anim Sci. 2007:85(13 Suppl):E45-62. doi:10.2527/jas.2006-508.

2. Hussein HS. Prevalence and pathogenicity of Shiga toxin-producing Escherichia coli in beef cattle and their products. J Anim Sci. 2007;85(13 Suppl):E63-72. doi:10.2527/jas.2006-421.

3. Smith JL, Fratamico PM, Gunther NW. Shiga toxin-producing Escherichia coli. Adv Appl Microbiol. 2014;86:145-97. doi:10.1016/b978-0-12-800262-9.00003-2.

4. Colello R, Etcheverría Al, Di Conza JA, Gutkind GO, Padola NL. Antibiotic resistance and integrons in Shiga toxin-producing Escherichia coli (STEC) Braz J Microbiol. 2015:46(1):1-5. doi:10.1590/s1517-838246120130698.

5. Pickering LK. Antimicrobial resistance among enteric pathogens. Semin Pediatr Infect Dis. 2004;15(2):71-7. doi:10.1053/j.spid.2004.01.009.

6. Murillo Llanes J, Varon J, Félix JSV, González-lbarra FP. Antimicrobial resistance of Escherichia coli in Mexico: how serious is the problem? J Infect Dev Ctries. 2012;6(2):126-31. doi:10.3855/jidc.1525.

7. Amábile-Cuevas CF. Antibiotic resistance in Mexico: a brief overview of the current status and its causes. J Infect Dev Ctries. 2010;4(3):126-31. doi:10.3855/jidc.427.

8. McManus PS, Stockwell VO, Sundin GW, Jones AL. Antibiotic use in plant agriculture. Annu Rev Phytopathol. 2002;40:443-65. doi:10.1146/annurev. phyto.40.120301.093927.

9. Van Boeckel TP, Brower C, Gilbert M, Grenfell BT, Levin SA, Robinson TP, et al. Global trends in antimicrobial use in food animals. Proc Natl Acad Sci U S A. 2015;112(18):5649-54. doi:10.1073/pnas.1503141112.

10. Bielaszewska M, Idelevich EA, Zhang W, Bauwens A, Schaumburg F, Mellmann A, et al. Effects of antibiotics on Shiga toxin 2 production and bacteriophage induction by epidemic Escherichia coli 0104:H4 strain. Antimicrob Agents Chemother. 2012;56(6):3277-82. doi:10.1128/aac.06315-11.

11. Smith KE, Wilker PR, Reiter PL, Hedican EB, Bender JB, Hedberg CW. Antibiotic treatment of Escherichia coli $\mathrm{O} 157$ infection and the risk of hemolytic uremic syndrome. Minnesota Pediatr Infect Dis J. 2012;31(1):37-41. doi:10.1097/INF. Ob013e31823096a8.

12. Zhang X, McDaniel AD, Wolf LE, Keusch GT, Waldor MK, Acheson DW Quinolone antibiotics induce Shiga toxin-encoding bacteriophages, toxin production, and death in mice. J Infect Dis. 2000;181(2):664-70. doi:10.1086/ 315239.

13. Corogeanu D, Willmes R, Wolke M, Plum G, Utermöhlen O, Krönke M. Therapeutic concentrations of antibiotics inhibit Shiga toxin release from enterohemorrhagic E. coli 0104:H4 from the 2011 German outbreak. BMC Microbiol. 2012;12:160. doi:10.1186/1471-2180-12-160.

14. Shiomi M, Togawa M, Fujita K, Murata R. Effect of early oral fluoroquinolones in hemorrhagic colitis due to Escherichia coli O157:H7. Pediatr Int. 1999;41(2):228-32. doi:10.1046/j.1442-200X.1999.4121038.x.

15. Ferens WA, Hovde CJ. Escherichia coli O157:H7: animal reservoir and sources of human infection. Foodborne Pathog Dis. 2011:8(4):465-87. doi:10.1089/ fpd.2010.0673.

16. Amézquita-López BA, Quiñones B, Cooley MB, Leon-Felix J, Castro-Del Campo N, Mandrell RE, et al. Genotypic analyses of Shiga toxin-producing Escherichia coli $\mathrm{O} 157$ and non-O157 recovered from feces of domestic animals on rural farms in Mexico. PLoS One. 2012;7(12):e51565. doi:10.1371/journal.pone. 0051565.

17. La Ragione RM, Best A, Woodward MJ, Wales AD. Escherichia coli O157:H7 colonization in small domestic ruminants. FEMS Microbiol Rev. 2009;33(2): 394-410. doi:10.1111/j.1574-6976.2008.00138.x.

18. Canizalez-Román A, González-Nuñez E, Vidal JE, Flores-Villaseñor H, León-Sicairos N. Prevalence and antibiotic resistance profiles of diarrheagenic Escherichia coli strains isolated from food items in Northwestern Mexico. Int J Food Microbiol. 2013;164(1):36-45. doi:10.1016/.i.jfoodmicro.2013.03.020.

19. Homedes N, Ugalde A. Mexican pharmacies and antibiotic consumption at the US-Mexico border. South Med Rev. 2012:5(2):9-19.

20. Wirtz VJ, Dreser A, Gonzales R. Trends in antibiotic utilization in eight Latin American countries, 1997-2007. Rev Panam Salud Publica. 2010;27(3):219-25. doi:10.1590/S1020-49892010000300009.

21. McGannon CM, Fuller CA, Weiss AA. Different classes of antibiotics differentially influence Shiga toxin production. Antimicrob Agents Chemother. 2010;54(9): 3790-8. doi:10.1128/aac.01783-09.

22. Amézquita-López BA, Quiñones B, Lee BG, Chaidez C. Virulence profiling of Shiga toxin-producing Escherichia coli recovered from domestic farm animals in Northwestern Mexico. Front Cell Infect Microbiol. 2014;4:7. doi:10. 3389/fcimb.2014.00007.

23. Clinical and Laboratory Standards Institute. Performance standards for antimicrobial susceptibility testing; 24th informational supplement. Wayne: CLSI Document M100-S24: 2014

24. Mehta CR, Patel NR. Algorithm 643. FEXACT: a FORTRAN subroutine for Fisher's exact test on unordered $1 \times c$ contingency tables. ACM Trans Math Softw. 1986;12(2):154-61. doi:10.1145/6497.214326.

25. Magiorakos AP, Srinivasan A, Carey RB, Carmeli Y, Falagas ME, Giske CG, et al. Multidrug-resistant, extensively drug-resistant and pandrug-resistant bacteria: An international expert proposal for interim standard definitions for acquired resistance. Clin Microbiol Infect. 2012;18(3):268-81. doi:10.1111/ j.1469-0691.2011.03570.x

\section{Submit your next manuscript to BioMed Central and we will help you at every step:}

- We accept pre-submission inquiries

- Our selector tool helps you to find the most relevant journal

- We provide round the clock customer support

- Convenient online submission

- Thorough peer review

- Inclusion in PubMed and all major indexing services

- Maximum visibility for your research

Submit your manuscript at www.biomedcentral.com/submit 